\title{
Routing the Flow of Sensory Signals Using Plastic Responses to Bursts and Isolated Spikes: Experiment and Theory
}

\author{
Jason W. Middleton, ${ }^{1} \mathrm{Na}$ Yu, ${ }^{2}$ André Longtin, ${ }^{2,3,4}$ and Leonard Maler ${ }^{3,4}$ \\ ${ }^{1}$ Department of Neurobiology, University of Pittsburgh School of Medicine, Pittsburgh, Pennsylvania 15213, ${ }^{2}$ Department of Physics, University of Ottawa, \\ Ottawa, Ontario K1N 6N5, Canada, and ${ }^{3}$ Department of Cellular and Molecular Medicine and ${ }^{4}$ Centre for Neural Dynamics, University of Ottawa, Ottawa, \\ Ontario K1H 8M5, Canada
}

\begin{abstract}
Processing complex sensory environments efficiently requires a diverse array of neural coding strategies. Neural codes relying on specific temporal patterning of action potentials may offer advantages over using solely spike rate codes. In particular, stimulus-dependent burst firing may carry additional information that isolated spikes do not. We use the well characterized electrosensory system of weakly electric fish to address how stimulus-dependent burst firing can determine the flow of information in feedforward neural circuits with different forms of short-term synaptic plasticity. Pyramidal cells in the electrosensory lateral line lobe burst in response to low-frequency, local (prey) signals. We show that the ability of pyramidal cells to code for local signals in the presence of additional high-frequency, global (communication) stimuli is uncompromised, while burst firing is reduced. We developed a bursting neuron model to understand how these effects, in particular noise-induced burst suppression, arise from interplay between incoming sensory signals and intrinsic neuronal dynamics. Finally, we examined how postsynaptic target populations preferentially respond to one of the two sensory mixtures (local vs local plus global) depending on whether the populations are in receipt of facilitating or depressing synapses. This form of feedforward neural architecture may allow for efficient information flow in the same neural pathway via either isolated or burst spikes, where the mechanisms by which stimuli are encoded are adaptable and sensitive to a diverse array of stimulus and contextual mixtures.
\end{abstract}

\section{Introduction}

Understanding how the brain processes information requires knowledge of the "neural code." Embedding information in spatiotemporal patterns of neural activity likely involves multiple mechanisms such as action potential propagation (Rieke et al., 1997), modulating synaptic strength distributions (Trussell, 1999; Mehta, 2001; Barrett and van Rossum, 2008), or dynamics of accessory networks such as glial or hemodynamic networks (Moore and Cao, 2008). Both firing rates and spike timing are important factors, determining the embodiment of information in spike trains (Knight, 1972; Mainen and Sejnowski, 1995; Panzeri et al., 2001).

Burst firing is an important feature of the neural code, given appropriate decoding schemes in downstream neural targets (Krahe and Gabbiani, 2004; Oswald et al., 2007). In addition to forming a specialized coding pathway, bursts may also effectively impact postsynaptic neurons in receipt of these spike packets (Thomson, 2000a). Synaptic short-term facilitation enhances membrane responses to bursts, thereby contributing to effective propagation of burst-encoded information. In contrast, single spikes more effectively transmit through depressing synapses (Lisman, 1997; Matveev and Wang, 2000). The question then

Received Sept. 6, 2010; revised Dec. 7, 2010; accepted Dec. 17, 2010.

This work was supported by a Canadian Institutes of Health Research (CIHR) grant (L.M., A.L.) and a Natural Sciences and Engineering Research Council of Canada grant (A.L.). J.W.M. is supported by a CIHR postdoctoral fellowship. We thank Anne-Marie Oswald for useful discussions and comments.

Correspondence should be addressed to Dr. Jason W. Middleton, Department of Neurobiology, University of Pittsburgh School of Medicine, 200 Lothrop Street, BST E1407, Pittsburgh, PA 15261. E-mail: jmiddlet@pitt.edu.

DOI:10.1523/JNEUROSCI.4672-10.2011

Copyright $\odot 2011$ the authors $\quad 0270-6474 / 11 / 312461-13 \$ 15.00 / 0$ arises whether these forms of plasticity are incorporated into different pathways devoted to different sensory cues and whether or not burst firing is dependent on stimulus, environmental, behavioral, or social contexts.

In the electrosensory system of the weakly electric fish Apteronotus leptorhynchus, burst firing is selective for certain stimulus classes (Oswald et al., 2004, 2007; Marsat et al., 2009). When stimuli impinge on the entire body (global stimulus), pyramidal cells of the electrosensory lateral line lobe (ELL) respond to highfrequency electrocommunication signals (Chacron et al., 2003). However, when stimuli are confined to receptive field (RF) centers (local stimulus), responses are coherent with low-frequency signals, typically arising from prey objects (Nelson and Maciver, 1999). Despite this paired dichotomy of spatial and temporal specificity, it is unclear how concurrent presentation of multiple stimuli (prey plus conspecifics) with different spatial and temporal properties affect electrosensory processing.

We recorded ELL pyramidal cells in vivo while stimulating with simultaneous global, high-frequency and local, lowfrequency electrosensory signals, thus mimicking naturally occurring temporally and spatially distributed signals. Spike patterning and stimulus coding were quantified under such naturalistic stimulus conditions. Coding measures were conserved across all stimulus conditions despite large changes in spike patterning. Most notably, global high frequencies reduced burst firing associated with local stimuli. We modeled pyramidal cell spiking activity to understand how spike pattern modulation arose from interplay between input currents and intrinsic dynamics. 
The ELL projects to the torus semicircularis (TS) and different downstream neurons show facilitation or depression in response to ELL stimulation (Fortune and Rose, 2000). We further propose a model in which different downstream target neurons receive either facilitating or depressing ELL synaptic input and therefore exhibit differential sensitivity to presynaptic burst versus isolated spike firing. We propose that depression and facilitation may enable routing of salient environmental information, arising from local sources (e.g., prey), to different downstream targets depending on electrosensory context (e.g., whether or not conspecifics were nearby).

\section{Materials and Methods}

Electrophysiology. Data from 10 adult A. leptorhynchus were used in this study. We recorded 30 E-type pyramidal cells and 12 I-type pyramidal cells. For surgical exposure of the ELL, fish were anesthetized (with Tricaine-S; Western Chemical). After surgery, fish were immobilized (pancuronium bromide; Sabex) and transferred to a tank $\left(28^{\circ} \mathrm{C}\right)$ where they were respired by a constant flow of oxygenated water through their mouth. Intracellular and extracellular recordings from ELL neurons and axons were made with borosilicate microelectrodes (70-140 M $\Omega$ ) filled with $3 \mathrm{~m} \mathrm{KAc} \mathrm{(for} \mathrm{intracellular} \mathrm{recordings)} \mathrm{or} \mathrm{tungsten} \mathrm{wire} \mathrm{electrodes}$ ( $\sim 1 \mathrm{M} \Omega$; for extracellular recordings; TM33C10; WPI). The recording electrodes were advanced into the ELL with a piezoelectric microdrive (Inchworm; IW-711; Burleigh). Cell type was identified based on electrode depth, baseline discharge statistics, and response to step changes in the electric organ discharge (EOD) amplitude as well as to sinusoidal and random EOD amplitude modulations (AMs); these responses are well characterized and successfully discriminate cell types (Saunders and Bastian, 1984; Bastian and Courtright, 1991). The ELL contains three topographic maps of the EOD-driven electroreceptors (Bell and Maler, 2005). The majority of recordings were, based on the topography of the ELL (Maler et al., 1991), from the centrolateral map, although we cannot exclude the possibility that a small subset were from the lateral map. In the centrolateral map, bursts encode only low-frequency local (prey-like) signals (Oswald et al., 2004; Marsat et al., 2009), whereas E-cells of the lateral ELL map use isolated spikes to encode high-frequency global communication signals and spike bursts encode a specific kind of transient communication signal (chirp) (Marsat et al., 2009). Recorded signals were amplified (Molecular Devices; for intracellular recordings), notch filtered at $60 \mathrm{~Hz}$ and the fish's EOD frequency (Ultra-Q Pro; Behringer), and stored on a desktop personal computer. Analysis was performed off-line by using Matlab software (The MathWorks). More complete animal husbandry and surgical techniques have been previously described (Hitschfeld et al., 2009). All experimental and surgical protocols were approved by the University of Ottawa Animal Care Committee and follow the guidelines established by the Canadian Council on Animal Care (1993).

Stimulation protocol. The EOD, unperturbed by the stimulus, was recorded between the head and tail of the fish using two vertical carbon rods (11 cm long, $8 \mathrm{~mm}$ diameter). A window discriminator (SD1; TDT) was used to detect the EOD zero-crossing times. TTL pulses occurring at the EOD zero-crossing times were used to drive a waveform generator (40 MS/s; Wavetek-Datron) in sine-wave-triggered mode, thereby creating a mimic of the fish's EOD. This EOD mimic was used as a carrier to deliver the stimulus as an AM of the fish's own EOD. The global stimulus was a $40-60 \mathrm{~Hz}$ bandpass Gaussian waveform, delivered across two electrodes (30-cm-long, 8-mm-diameter carbon rods) placed $10 \mathrm{~cm}$ away on either side of the fish. This stimulus was designed to mimic the sensory input from two or more neighboring fish (Middleton et al., 2006; Stamper et al., 2010). The local stimulus, delivered via a small stimulus dipole ( $\sim 2-5 \mathrm{~mm}$ spacing, $2-3 \mathrm{~mm}$ away from the fish), was a $0-20 \mathrm{~Hz}$ low-pass Gaussian waveform. This stimulus was designed to mimic preyrelated electrosensory signals (Nelson and Maciver, 1999). Both stimuli were attenuated (PA4; TDT), multiplied with the EOD mimic, and delivered by their respective dipoles to result in amplitude modulations without concurrent frequency modulations. Recorded EOD voltages (head-tail and transverse) were amplified and low-pass filtered at $5 \mathrm{kHz}$
(2015F; Intronix). Global and local stimulus intensities were adjusted so that they were both in the $5-15 \%$ contrast range and that they each resulted in similar sinusoidal rate modulations when presented alone. The extracellular potential, the EOD, the transdermal potential, and the attenuated stimulus were digitized at $20 \mathrm{kHz}$ with a data acquisition interface (Power 1401; Cambridge Electronics Design) and recorded using Spike2 software (Cambridge Electronics Design). Spike time detection, stimulus generation, and analysis of the data were performed offline using Spike2 and Matlab software (The MathWorks).

Data analysis. Action potentials were recorded in response to repeated presentations of 5-s-long realizations of local stimuli, global stimuli, or a combination of both. Spike train statistics and stimulus response metrics (spike-triggered average and coherence) were calculated using spikes from all stimulation trials. Spike-triggered averages (STAs) were obtained by averaging the (normalized) stimulus waveform within a short time window surrounding recording spikes, in other words, the following:

$$
\operatorname{STA}(t)=\frac{1}{n} \sum_{i=1}^{n} s\left(t-t_{i}\right),
$$

where $s(t)$ is the stimulus waveform and $\left[t_{i}\right]$ is the set of $n$ recorded spike times. The coherence between stimulus, $s(t)$, and spike train, $x(t)$, measures frequency-specific correlation of the two signals as follows:

$$
C_{s x}(f)=\frac{\left|S_{s x}(f)\right|^{2}}{S_{s s}(f) S_{x x}(f)},
$$

where $S$ denotes either the auto-power spectral density or crossspectral density, depending on the subscripts (Gabbiani and Koch, 1998). The stimuli, $s(t)$, are the random amplitude modulations (RAMs) of the EOD that we delivered to the fish (as described above in Stimulation protocol). Therefore, when coherence is calculated, $s(t)$ represents either local, global, or local plus global stimuli as specified in the pertinent text. The spike trains, $x(t)$, are either the full spike train, spike trains composed of bursts, or spike trains composed of isolated spikes, also specified when necessary. Unless otherwise stated, significance was tested by one-way ANOVA followed by Tukey's honestly significant difference test.

Modeling. The model we used was based on a previous model used to reproduce burst firing observed in ELL pyramidal cells in vitro (Doiron et al., 2007). This model is a reduction of previous dynamical models (Doiron et al., 2002; Laing et al., 2003; Fernandez et al., 2005) that are themselves derived from a more realistic model based on the biophysics of pyramidal cells (Doiron et al., 2001). The reduced model has been shown to successfully replicate the emission of isolated and burst spikes evoked by broadband current injection (Doiron et al., 2007; Oswald et al., 2007). The model dynamics are as follows:

$$
C \frac{d v}{d t}=b-g V+A p\left(t-\tau_{\mathrm{DAP}}\right) \times\left(t-\tau_{\mathrm{DAP}}\right)+l_{\text {stim }}(t)+l_{\text {noise }}(t),
$$

$$
\begin{gathered}
\frac{d x}{d t}=y, \\
\frac{d y}{d t}=-\alpha^{2} x-2 \alpha y+\alpha^{2} \sum_{m} \delta\left(t-t_{m}\right) \theta\left(t-t_{m-1}-r\right), \\
\tau_{p} \frac{d p}{d t}=p_{\infty}-p+B p^{2} \sum_{m} \delta\left(t-t_{m}\right) \theta\left(t-t_{m-1}-r\right), \\
\tau_{r} \frac{d r}{d t}=r_{\infty}-r+c r \sum_{m} \delta\left(t-t_{m}\right) \theta\left(t-t_{m-1}-r\right),
\end{gathered}
$$

where $V$ is the transmembrane potential, $b$ is an input bias current, and $g$ is a passive leak conductance. In the absence of any other currents, $b$ and $g$ set the steady-state resting potential of the membrane: $V_{\text {rest }}=b / g . x$ and 
$y$ are the amplitude and derivative, respectively, of the dendritic current variable. $x$ is scaled by $A p$, where $A$ is the maximal current and $p$ is a dynamic potentiation factor that promotes bursting (Lemon and Turner, 2000). The variable, $r$, is a dynamic, spike-driven refractory period. $p$ and $r$ are updated at every spike time such that $p \rightarrow p+B p^{2}$ and $r \rightarrow r+c r$. However, if the relative spike time, since the previous one, occurs within the refractory period, $r$, the variables, $y, p$, and $r$ are not updated and relax back to their baseline values. This model is a modification of the basic "leaky integrate-and-fire" neuron model (Tuckwell, 1988). Input currents are integrated, and if the membrane voltage is sufficiently depolarized and reaches spike threshold, $v_{\text {thresh }}$, the membrane voltage is immediately set to a reset voltage, $v_{\text {reset }}$. Unless otherwise specified, the parameter values used are as follows: $C=0.25 \mu \mathrm{F} / \mathrm{cm}^{2}, b=0.89 \mu \mathrm{A} /$ $\mathrm{cm}^{2}, g=0.0655 \mathrm{mS} / \mathrm{cm}^{2}, A=1.1 \mu \mathrm{A} / \mathrm{cm}^{2}, \tau_{\mathrm{DAP}}=2 \mathrm{~ms}, \alpha=1.5 \mathrm{~ms}^{-1}$, $\tau_{p}=5 \mathrm{~ms}, p_{\infty}=0.4, B=0.3, \tau_{r}=5 \mathrm{~ms}, r_{\infty}=4 \mathrm{~ms}, c=0.4, v_{\text {reset }}=0 \mathrm{mV}$, and $v_{\text {thresh }}=15 \mathrm{mV}$.

Even though the model does not directly incorporate spatially extended input, the external stimulus input, $I_{\text {stim }}=\sigma_{L} \eta_{L}+\sigma_{G} \eta_{G}$, has both local and global components representing the net current input at the soma from both peripheral sources. Both stimuli are bandpass Gaussian noise processes with bandwidths of $0-20$ and $40-60 \mathrm{~Hz}$ for local and global stimuli, respectively. The stimulus amplitudes are $\sigma_{L}=0.065$ $\mu \mathrm{A} / \mathrm{cm}^{2}$ and $\sigma_{G}=0.09 \mu \mathrm{A} / \mathrm{cm}^{2}$ unless otherwise stated. The intrinsic noise current, $I_{\text {noise }}$, is a Gaussian white noise process with a low-pass cutoff of $200 \mathrm{~Hz}$ and SD of $0.1 \mu \mathrm{A} / \mathrm{cm}^{2}$.

ELL pyramidal cells project to a midbrain region (TS) similar to the inferior colliculus of mammals (Bell and Maler, 2005). Previous in vivo studies have shown that ELL-to-TS synapses exhibit both short-term facilitation and depression (Fortune and Rose, 2000, 2001). We used a phenomenological model of plastic synapses that has been successfully used to quantitatively reproduce the dynamics of feedback plasticity in the ELL (Lewis and Maler, 2001). The strength of postsynaptic conductance is determined by two plasticity variables, $F$ and $D$, for facilitation and depression, respectively. The dynamics of the postsynaptic conductance is as follows:

$$
\frac{d G}{d t}=\frac{G}{\tau_{G}}+a \cdot F \cdot D \sum_{m} \delta\left(t-t_{m}\right),
$$

where $t_{m}$ are presynaptic spike times. For the purpose of our study, we will be studying synapses that are either facilitating or depressing, so that when one variable is dynamic the other is held constant at 1 . The dynamics for the plasticity variables are as follows:

$$
\begin{aligned}
& \frac{d F}{d t}=\frac{F_{0}-F}{\tau_{F}}, \\
& \frac{d D}{d t}=\frac{1-D}{\tau_{D}} .
\end{aligned}
$$

Both variables obey fractional update rules at the times of presynaptic spikes that capture the effects of either depletion of synaptic resources (Lewis and Maler, 2001) or saturation of presynaptic calcium (Tsodyks et al., 1998): $F \rightarrow F+\Delta_{F}(1-F)$ and $D \rightarrow \Delta_{D} D$ for facilitation and depression, respectively. $F_{0}$ represents the baseline probability of release of facilitating synapses in absence of any spiking history, whereas the baseline probability of release of depressing synapses is 1 (Lewis and Maler, 2001). A small value of $F_{0}$ ensures that the strength of facilitating synapses increases with repetitive stimulation. The parameter values used are $\tau_{F}=$ $110 \mathrm{~ms}, \tau_{D}=45 \mathrm{~ms}, \tau_{G}=3 \mathrm{~ms}, F_{0}=0.1, \Delta F=0.1, \Delta_{D}=0.6$, and $a=$ 0.065 (for depression) or 0.2 (for facilitation).

The postsynaptic membrane dynamics, putatively representing a target midbrain cell, are described by the following equation for generic leaky integrate-and-fire dynamics:

$$
C_{m} \frac{d V_{m}}{d t}=-g_{\text {leak }}\left(V_{m}-V_{L}\right)-g_{\text {syn }} \cdot G(t)\left(V_{m}-V_{E}\right),
$$

with spike threshold and spike reset values of $V_{\text {thresh }}=-45 \mathrm{mV}$ and $V_{\text {reset }}=-70 \mathrm{mV}$, respectively. The parameters used for this neural model are as follows: $C_{m}=1 \mu \mathrm{F} / \mathrm{cm}^{2}, V_{L}=-70 \mathrm{mV}, g_{\text {leak }}=1 \mathrm{mS} / \mathrm{cm}^{2}$, $g_{\text {syn }}=18 \mathrm{mS} / \mathrm{cm}^{2}$, and $V_{E}=0 \mathrm{mV}$.

\section{Results}

\section{Local low-frequency and global high-frequency stimuli} differentially modulate burst firing: experiments

Electrosensory stimuli were presented in one of three configurations (Fig. 1a): local (L), global (G), or simultaneous local and global stimulation $(\mathrm{L}+\mathrm{G})$. Local amplitude modulations, covering the RF center of a pyramidal cell, were delivered by a small dipole near the fish's skin. The local stimuli waveforms were composed of low-frequency fluctuations $(0-20 \mathrm{~Hz})$, motivated by reports that small prey-like objects result in slow, local amplitude modulations (Nelson and Maciver, 1999). An electrode pair placed further away on both sides of the fish's body was used to deliver global stimulus. This stimulus consisted of highfrequency amplitude modulations $(40-60 \mathrm{~Hz})$, consistent with the spatiotemporal content of electrosensory signals from nearby conspecifics (Tan et al., 2005; Ramcharitar et al., 2006; Stamper et al., 2010).

ELL pyramidal cells consist of two major categories: E- and I-cells (Bell and Maler, 2005). E-cells respond to increases in EOD intensity within the receptive field center of the cell (Saunders and Bastian, 1984; Bastian et al., 2002) as would result naturally from the presence of prey. Some E-cells (centrolateral and lateral maps) also respond to global, high-frequency signals associated with electrocommunication. I-cells respond to decreases in EOD intensity within the receptive field center of the cell as would result naturally from the presence of small nonconductors (rocks). The E-cells showed the most prominent changes in firing characteristics associated with global plus local input and so the results presented below are confined to E-cells unless otherwise stated.

The slow features of local stimuli are effective in eliciting bursts in centrolateral map E-type pyramidal cells (Fig. 1b, top), consistent with previous in vitro (Oswald et al., 2004, 2007) and in vivo (Marsat et al., 2009) studies. Burst firing is greatly diminished when a high-frequency global stimulus is instead delivered (Fig. 1b, middle) and responses consisted mainly of single spikes. Simultaneous delivery of $\mathrm{L}$ and $\mathrm{G}$ stimuli results in an intermediate level of burst activity_-higher than with $\mathrm{G}$ alone (Fig. 1b, bottom) but much less than with $\mathrm{L}$ alone.

The population-averaged behavior of this burst firing can be seen in the interspike interval histograms (ISIHs). During local stimulation, a large peak is present at short interspike intervals (ISIs) $(<10 \mathrm{~ms}$ ) (ISIH-L) (Fig. $2 a)$. This ISIH structure is a hallmark of burst firing (Krahe and Gabbiani, 2004) and may additionally have a second, well defined mode at larger ISIs. The peak at short ISIs was reduced during global stimulation alone; rather, there was a well defined mode at $\sim 20 \mathrm{~ms}$, the mean timescale of the $40-60 \mathrm{~Hz}$ temporal frequency content of the global stimulus. Local and global stimulation together resulted in an ISIH that was similar to the G-alone ISIH, but with a marginally higher rate of short ISIs.

To further quantify burst responses, we identified specific spike sequences as bursts if consecutive ISIs between the spikes were less than the "ISI threshold." The threshold was chosen to be the ISI value where the ISIH-L and ISIH-G crossed; in our data, the value was $14.5 \mathrm{~ms}$. The selection of burst threshold is somewhat arbitrary and could be based on other criteria (e.g., the interval at which ISIHs deviate from a Poisson distribution) (Bas- 
tian and Nguyenkim, 2001). However, small perturbations of the cutoff value we used did not qualitatively affect our results (data not shown). Under all stimulus conditions, the number of spikes per burst remains unchanged $(\mathrm{L}, 2.56 \pm 0.07 ; \mathrm{G}$, $2.52 \pm 0.11 ; \mathrm{L}+\mathrm{G}, 2.48 \pm 0.06 ; p=$ 0.3294, one-way ANOVA) (Fig. 2b). However, the burst rate is higher with local (L) $(3.2 \pm 0.4 \mathrm{~Hz})$ than with either global $(\mathrm{G})\left(0.6 \pm 0.1 \mathrm{~Hz} ; p<10^{-3}\right)$ or local plus global $(\mathrm{L}+\mathrm{G})(1.3 \pm 0.2 \mathrm{~Hz} ; p<$ $10^{-3}$ ) (Fig. 2b) stimulation. The population average firing rates are not significantly different under the different stimulus conditions: $26.9 \pm 2.0 \mathrm{~Hz}(\mathrm{~L})$, $23.5 \pm 1.6 \mathrm{~Hz}(\mathrm{G})$, and $24.9 \pm 1.6 \mathrm{~Hz}$ $(\mathrm{L}+\mathrm{G})(p=0.3582$, one-way ANOVA $)$; therefore, any stimulus dependence of coding properties is not likely attributable to firing rate effects. Similar qualitative effects are seen for nonbasilar, or I-type, pyramidal cells (supplemental Fig. 1, available at www.jneurosci.org as supplemental material); however, since the magnitude of the ISIH shaping is relatively smaller in I-cells, we will focus solely on E-type pyramidal cells.

STAs were analyzed with respect to local or global stimulus components separately (see Materials and Methods). Both global and local stimulus waveform STAs are very similar under all stimulus conditions (Fig. 2c). The coherence between pyramidal spike trains and the $40-60 \mathrm{~Hz}$ signal is the same with either $G$ or $L+G$ stimulus conditions (Fig. 2d), indicating that the $40-60 \mathrm{~Hz}$ signal effectively entrains the pyramidal cell spike train temporal patterning (mean coherence over $40-60 \mathrm{~Hz}$ is $\sim 0.4$ in both cases). Local stimulation also strongly modulates the pyramidal cell spike trains (mean coherence over $0-20 \mathrm{~Hz}$ is $\sim 0.4$ ). Concurrent delivery of global and local stimuli only slightly decreases the low-frequency component of its coherence (Fig. $2 d$ ) (mean coherence over $0-20 \mathrm{~Hz}$ is reduced to $\sim 0.3$ ) despite a large reduction in burst firing. Together, the small relative changes in both $(0-20 \mathrm{~Hz})$ STA and coherence imply that the ability of pyramidal cells to encode local low-frequency electrosensory signals remains relatively unchanged, whereas temporal spiking patterns are considerably altered with the linear addition of a high-frequency global stimulus.

E-type pyramidal cells exhibit a broad range of spontaneous and stimulusevoked firing properties. This variability can be primarily explained by differences in dendritic morphology (Bastian and a

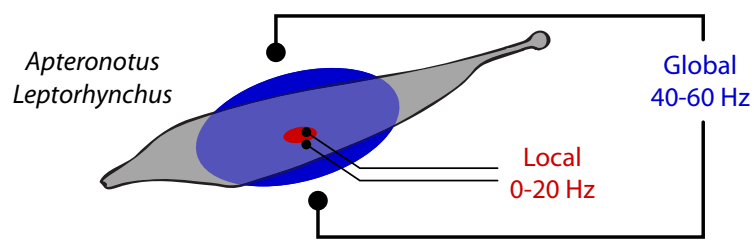

b

Local

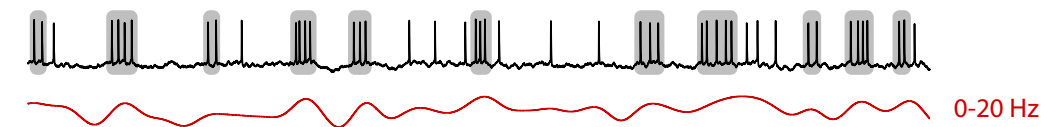

Global
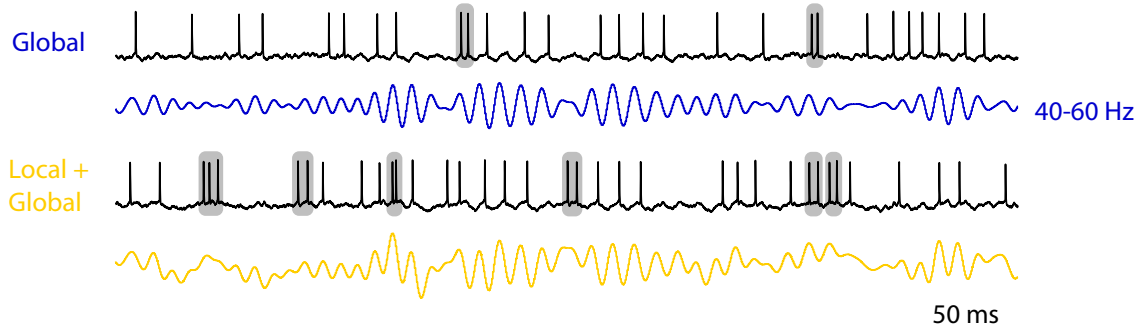

Figure 1. Mixed high- and low-frequency stimuli determine firing patterns of electrosensory pyramidal cells. $\boldsymbol{a}$, Weakly electric fish $A$. leptorhynchus were presented electrosensory stimuli, in the form of RAMs from one of two sources. Lowfrequency $(0-20 \mathrm{~Hz})$ RAMs were delivered via a small dipole centered on the $\mathrm{RF}$ center of a pyramidal cell resulting in local EOD modulations (red), whereas high-frequency $(40-60 \mathrm{~Hz})$ RAMs were delivered via dipoles $20 \mathrm{~cm}$ apart, centered on the head-tail axis of the fish. These dipoles generated global EOD modulations (blue) spanning most of the body. $\boldsymbol{b}$, Local, low-frequency RAMs elicit burst firing in an E-type pyramidal cell (top). These bursts coincide with slow, positive-going (depolarizing) features of the stimulus. The same pyramidal cell produces mostly isolated spikes when presented with global, high-frequency RAMs (middle). Simultaneous delivery of both stimuli results in more burst firing than with global alone, but much less than with local stimuli (bottom). a

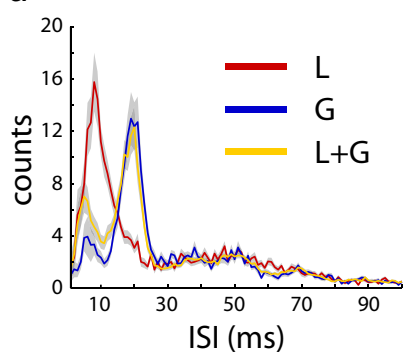

C
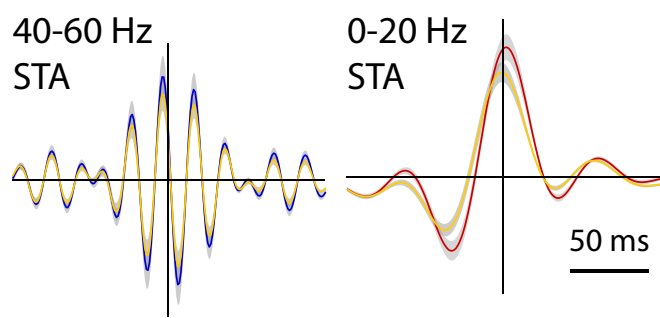

b

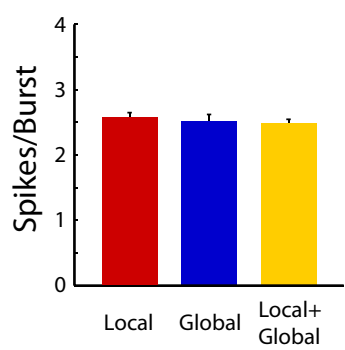

Figure 2. High-frequency stimuli reduce burst firing in pyramidal cells while preserving the encoding of low-frequency local signals. $\boldsymbol{a}$, The ISIH for local stimuli (L) (red) exhibits a large mode at short ISIs, indicative of burst firing. When global stimuli (G) (blue) are presented a prominent mode centered at $20 \mathrm{~ms}$ is apparent. With local and global stimuli together ( $L+G)$ (yellow), the ISIH is more similar to the global ISIH. $\boldsymbol{b}$, The number of spikes per burst was similar under all three stimulus conditions. The burst rate under local stimulation is higher than either global or local plus global. c, STAs for either the global (left) or local (right) stimulus components were similar under all three stimulus conditions ( $L$, red; $G$, blue; $L+G$, yellow). The vertical axes represent the reference spike time. $\boldsymbol{d}$, The coherence between spike trains and local stimulus is slightly smaller under $L+G$ conditions compared with $L$ alone. The coherence with global stimulus is the same under either $G$ or $L+G$ conditions. The error bars and gray shaded regions indicate SEM. 

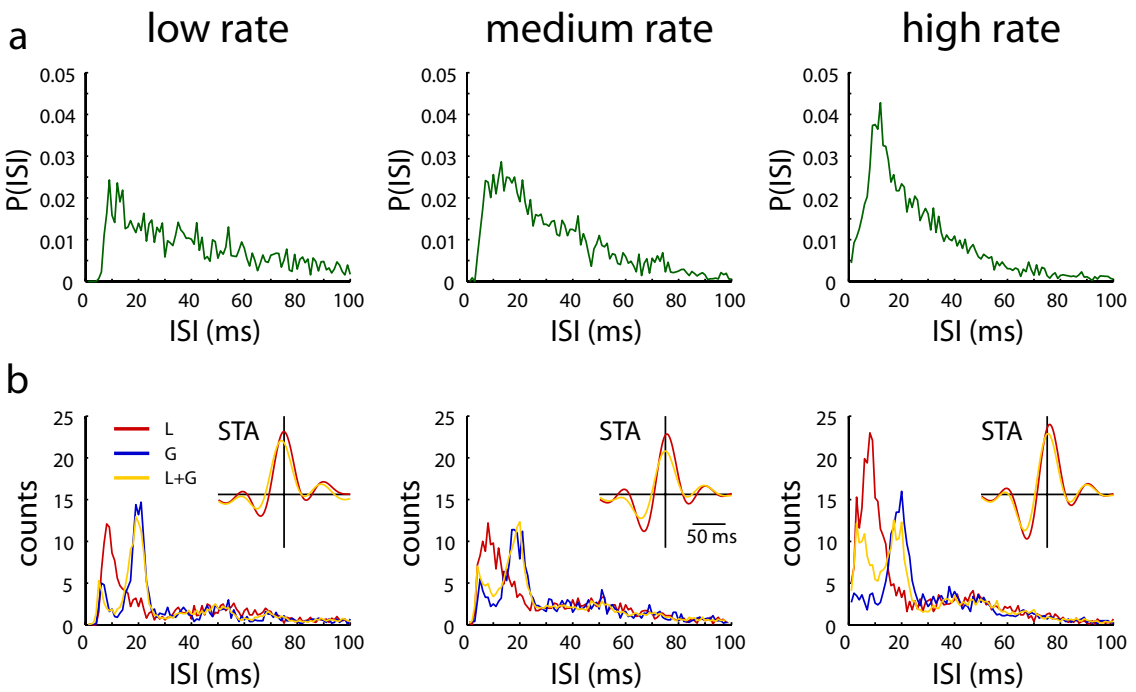

Figure 3. Stimulus-dependent burst firing in subtypes of ELL basilar pyramidal cells. Cells were divided into three groups according to their mean spontaneous firing rates: low $\left(r_{\text {spont }}<21 \mathrm{~Hz}\right)$, medium $\left(21 \mathrm{~Hz} \leq r_{\text {spont }}<29 \mathrm{~Hz}\right)$, and high $\left(r_{\text {spont }} \geq 29\right.$ $\mathrm{Hz}$ ), putatively corresponding to superficial, intermediate, and deep pyramidal cells, respectively. $\boldsymbol{a}$, Spontaneous ISIHs for (from left to right) low, medium, and high firing rate groups. $\boldsymbol{b}$, ISIHs under local (red), global (blue), and local plus global (yellow) stimulus conditions for (from left to right) low, medium, and high firing rate groups. The insets show local (red) and local plus global (yellow) STAs of the $0-20 \mathrm{~Hz}$ stimulus component.

Nguyenkim, 2001). To examine whether these heterogeneities affect observed stimulus-dependent burst structure, we divided our original data set into three equally sized subsets $(n=10$ each) based on their mean spontaneous firing rates, $r_{\text {spont }}$. These groups, labeled low, medium, and high firing rate groups, putatively correspond to superficial, intermediate, and deep pyramidal cells, respectively (Bastian and Courtright, 1991; Bastian and Nguyenkim, 2001; Chacron et al., 2005).

The spontaneous ISIHs are characterized by progressively less skewed distribution containing smaller ISIs, going from low rate to high rate subtypes (Fig. 3a). All cell types become more "bursty" (an increase in the mode at small ISIs) with local, lowfrequency electrosensory stimulation (Fig. 3b). This large burst mode is effectively reduced by concurrent delivery of global stimulation (Fig. 3b). Despite the presence of small quantitative differences in the ISIH structure, all pyramidal cell subtypes qualitatively exhibit local stimulus-induced bursting that is inhibited by concurrent delivery of global, high-frequency electrosensory stimulation. For this reason, all following analysis and modeling will focus on the population average of the entire data set.

Burst firing in pyramidal cells encodes low-frequency stimuli as previously shown in vitro (Oswald et al., 2004) and in vivo (Marsat et al., 2009). The ability for pyramidal cells to encode local signals implies that slow rate modulations, "riding on" the fine temporal structure inherited from the global high-frequency stimulus, are coherent with slow stimulus features contained within the local signal. It was previously shown that the higher order features of $40-60 \mathrm{~Hz}$ signals, namely stimulus envelopes (Middleton et al., 2006), are encoded by E-cells spiking activity. For a $40-60 \mathrm{~Hz}$ stimulus, the corresponding envelope has power in the $0-20 \mathrm{~Hz}$ range (Middleton et al., 2007). The envelope response magnitudes, for both STA and coherence, in this range are typically much smaller than those to direct local, $0-20 \mathrm{~Hz}$ signals. The relative magnitude reduction of these response measures is large with the concurrent delivery of local stimulus (supplemental Fig. 2, available at www.jneurosci.org as supplemental material). If the envelope does represent a behaviorally relevant signal as suggested by recent recordings from TS (Vonderschen and Chacron, 2009), the information pertaining to it is presumably conveyed to TS neurons by E-cells with receptive fields covering other regions of the fish's body that are subject only to the global stimulus. The envelope response in these cells would thus not be masked by the local stimulus.

\section{Conditional backpropagation-induced burst firing depends on stimulus frequencies: theory}

We investigated the dependence of burst firing on the temporal frequency content of incoming stimuli using a modified pyramidal cell model (Doiron et al., 2007). This single compartment model incorporates the effect of an additional dendritic compartment through the delayed feedback of a stereotyped waveform (Fig. 4a) (see Materials and Methods). This captures the effect of backpropagating spikes resulting in depolarizing afterpotentials (DAPs) at the soma, consistent with both in vitro (Noonan et al., 2003; Ellis et al., 2007) and in vivo (Marsat et al., 2009) data. We modeled a conditional burst mechanism by incorporating slow dynamics that account for the potentiation of DAPs and dendritic refractory period. This produces both spike time acceleration and subsequent burst termination when ISIs become shorter than the refractory period (Fig. $4 a$, right). In addition, leak conductance values in the model were changed for each stimulus condition to obtain qualitatively similar firing rates to those seen in data. The values used are $g=0.0655 \mathrm{mS} / \mathrm{cm}^{2}$ for $\mathrm{L}, g=0.0675 \mathrm{mS} / \mathrm{cm}^{2}$ for $\mathrm{G}$, and $g=0.069 \mathrm{mS} / \mathrm{cm}^{2}$ for $\mathrm{L}+\mathrm{G}$ stimulus conditions. Varying leak conductance values is also consistent with the observation that pyramidal cell membrane conductance presumably increases under global stimulation caused by parallel fiber feedback (Nelson, 1994).

When the model is driven with stimuli corresponding to bandwidths used for local $(0-20 \mathrm{~Hz})$ and global $(40-60 \mathrm{~Hz})$ stimuli, the burst firing of the output spike train is stimulus specific. With "local" stimulation, the model predominantly exhibited burst firing patterns (Fig. 4b). With "global" stimulation, burst firing was mostly diminished, and with a linear sum of the two signals, it was slightly more prevalent than with global stimulus alone, but much less than with local stimulus. As expected, these different levels of bursting can be seen in the model ISIHs (Fig. 4c), showing very good qualitative agreement with observed data. The model also reproduces a very similar set of burst properties to that observed in the data. Although the number of spikes per burst changes slightly under different stimulus conditions, the burst rate shows the same qualitative ordering seen in experimental data (i.e., $\mathrm{L}>\mathrm{L}+\mathrm{G}>\mathrm{G}$ ) (Fig. $4 d$ ). The local signal STA is only slightly different than with concurrent global inputs (Fig. $4 e$ ), as seen in the data (Fig. 2). Finally, the model also reproduces the data in that, under $\mathrm{L}+\mathrm{G}$ conditions, it encodes both the $40-60 \mathrm{~Hz}$ and the $0-20 \mathrm{~Hz}$ signals albeit with lower levels of coherence than those seen under either L-alone or G-alone stimulation conditions (Fig. $4 f$ ).

To understand the mechanism underlying stimulus frequency dependence of burst firing, we calculated two parameter bifurca- 
tion sets for the deterministic model without fluctuating inputs (Fig. 5a). These bifurcations delineate different parameter regimens corresponding to qualitatively different firing patterns. The conductance-bias $(g, b)$ pair values used for the different stimulus conditions lie in the quiescent regimen and are separated from the bursting regimen, along the $b$-axis, by a small region of periodic firing. How slow signals promote burst firing can be intuitively understood by treating the slow current fluctuations as a proxy for stationary changes in the bias. In this quasi-static description, low-frequency depolarizing phases of the stimulus slowly shift the fixed point to the right, along the $b$-axis, into the bursting regimen. In the case of sufficiently long depolarizations arising from low-frequency stimuli, bursts are terminated by the dendritic refractory period. However, stimulus-induced termination by negative-going components of high-frequency stimuli also occur (Doiron et al., 2007). Thus, efficacy of burst generation by time-varying stimulus will depend on the stimulus temporal frequency content. We constructed an index to quantify the relative "burstiness" of a spike train, based on ISIH criteria. This burst index (BI) is obtained by dividing the integral of the ISIH below the ISI threshold by the integral of the ISIH above threshold. Based on the ISIHs shown in Figures $2-4$, it is clear that $0-20 \mathrm{~Hz}$ will result in a larger BI than $40-60 \mathrm{~Hz}$ stimulus frequencies. The specific temporal frequency content dependency of the BI is shown in Figure $5 b$. Low-frequency, narrow-bandwidth stimuli promote bursting, whereas increasing either center frequency or bandwidth decrease BI. The second region apparently exhibiting large burst index (high center frequency, low bandwidth) arises from very irregular, nonphysiological firing patterns. In this parameter regimen, the model receives high-frequency narrowband fluctuating inputs. The impact of this high-frequency stimulus on membrane variability is attenuated because of passive low-pass membrane properties. The narrowband nature of the stimulus results in a slowly varying instantaneous amplitude of the narrowband oscillation. As a result of these two effects, the model is very rarely depolarized sufficiently enough to spike, but when it does it typically emits more than one spike.

This concept of "burst excitability" was introduced to describe how transient inputs with certain properties elicit bursts in a deterministic neuron model with an intrinsic burst mechanism (Laing et al., 2003). The burst threshold (i.e., minimal duration of a current pulse needed to excite a burst) was found to scale inversely with the pulse height. Depolarizing phases of a timevarying stimulus will have similar effects as a square-wave current pulse. Namely, broader phases, such as contained in L stimuli

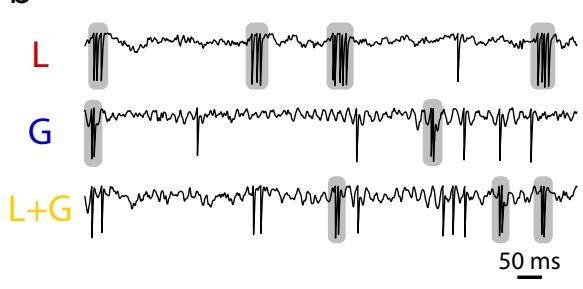

C

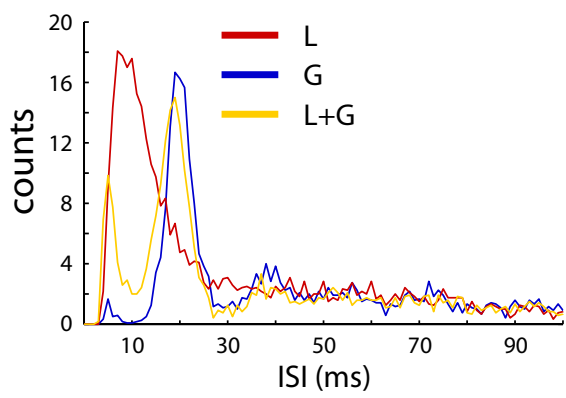

d
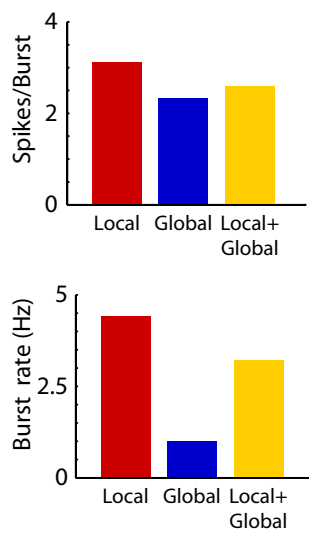

e

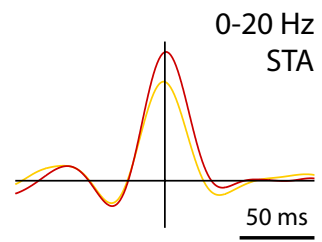

f

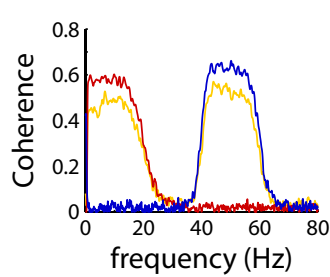

Figure 4. Burst firing, resulting from DAPs, is reduced by high-frequency stimuli in a pyramidal cell model. $\boldsymbol{a}$, A schematic of the ELL pyramidal cell model (see Materials and Methods) illustrates the effect of delayed feedback from the dendrite to the soma (left). One burst cycle of the deterministic model results (right) shows the dendritic DAP, somatic voltage, and the output spike 列 (Fig. 2a), result in a large mode at higher ISI values ( $\sim 20 \mathrm{~ms}$; blue line). When high-frequency inputs are delivered with low-frequency inputs, the frequency of short ISIs (burst-like events) is mostly reduced (yellow line). $\boldsymbol{d}$, The number of spikes per burst that the model produces is similar under all stimulus conditions (left), whereas more bursts were produced with local stimulus (right). The remains similar under both $\mathrm{L}$ and $\mathrm{L}+\mathrm{G}$ stimulus conditions. $\boldsymbol{f}$, Stimulus response coherences qualitatively reproduce those seen in data. Low-frequency coherence $(0-20 \mathrm{~Hz})$ is slightly smaller with concurrent global stimulation.

(Fig. $5 c$, top), will promote bursting more than the brief phases more frequently seen in $\mathrm{G}$ or $\mathrm{L}+\mathrm{G}$ stimulus conditions (Fig. $5 c$, middle and bottom). We numerically obtained the burst threshold using smooth pulses (half-sine waves) and show that our model also has an inverse duration-height scaling law and that the average depolarization height and width for local stimuli are above the burst threshold curve, whereas the average height and width points for local and local plus global are below this curve (Fig. 5d).

In simple terms, our model suggests that global highfrequency signal components suppress bursting by transiently interrupting the long depolarizations caused by low-frequency signals, thereby preventing activation of the back and forth burst mechanism. However, the mean depolarization attributable to positive phases of low-frequency AMs is still increased despite 
a

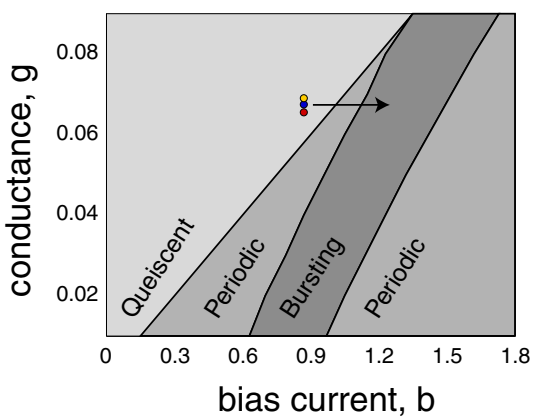

C

d: Pulse duration

h: Pulse height

$\mathrm{L}$

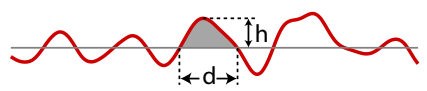

G
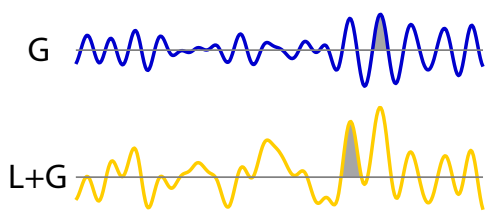

$\mathrm{b}$

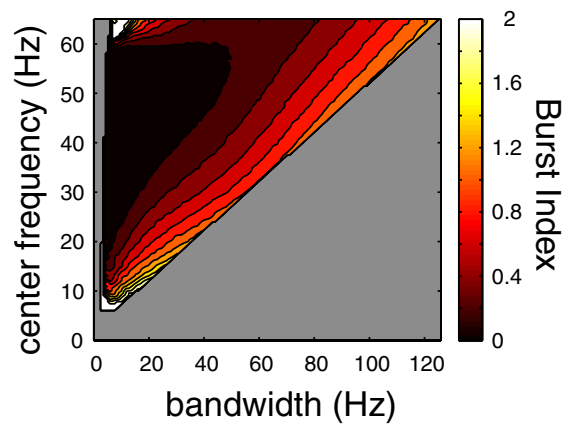

d

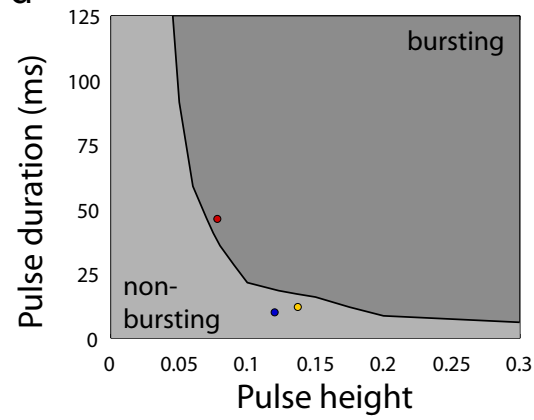

Figure 5. Low-frequency inputs promote burst excitability, whereas additional high-frequency inputs interfere with excitability. $\boldsymbol{a}$, The two-parameter bifurcation set outlines the different firing regimens of our model. The conductance- bias pairs used for $L$ (red), $G$ (blue), and $L+G$ (yellow) lie in the quiescent regimen, separated from the bursting regimen by a narrow region of periodic firing. $\boldsymbol{b}, \mathrm{A}$ BI (the ratio of low to high ISIH counts) shows that low-frequency, narrowband stimuli are most effective at producing bursts. c, Depolarizing phases of local stimuli (top, shaded area) are broader than depolarizing phases of global (middle) or local plus global (bottom) stimuli. $\boldsymbol{d}$, Burst threshold was numerically calculated by giving the model neuron half-sine wave current pulses of a given duration (or width) and height. The parameter space was divided into two regions: (1) dark gray indicates the region of parameter pairs producing sufficiently strong pulses to evoke at least one burst, and (2) light gray indicates the region where pulses do not evoke bursts. The threshold of pulse duration (dividing bursting and nonbursting regions; black line) scales inversely with pulse height. To see how our stimulus statistics relate to this threshold, we calculated the average height and width of the portion of the stimulus above zero ( $\boldsymbol{c}$, gray regions). The average duration and height of depolarizing phases of local stimuli place it above the burst threshold curve (dark gray region), whereas global and local plus global lie below the curve (light gray region).

these transient interruptions; this explains why isolated spikes can still encode these low-frequency local signals.

\section{Burst and isolated spike firing scale with global stimulus intensity}

Our results so far suggest that both temporal coding and temporal encoding are at play in our system (Theunissen and Miller, 1995). The former implies that the temporal structure of the stimulus (AMs in our case) is reflected in a similar temporal modulation of spiking probability; the input-output coherence is a measure of the linearity of this relation. However, temporal encoding refers to the case wherein a temporal pattern of spikes (such as bursts) occurs reliably in the presence of a particular aspect of the stimulus. We have shown how burst firing depends on the temporal frequency content of the AM stimuli. We now show how the coding of stimulus-related information is shared between burst spikes and isolated spikes. As burst firing is suppressed by global high-frequency AMs, we show that this coding also scales proportionally to continuous variations in the intensity of the global stimulus. To assess this dependence, we recorded from a subset of neurons $(N=15$ of 30$)$ to which global stimuli were delivered at different intensities $( \pm 4 \mathrm{~dB})$ than the original global intensity. Higher or lower global intensities either decreased or increased, respectively, the proportion of short ISIs

observed (Fig. 6a). Despite these changes in burst proportion, there was relatively little change in the STA of the concurrent low-frequency local signal (Fig. 6b).

To further investigate this issue, we analyzed the spectral properties of spike trains comprised of either bursts or isolated spikes and, in particular, how they depended on the intensity of the global stimulus. The coherence of the original spike train (all spikes) to the local signal decreases with increasing global intensity (Fig. 6c, top). Accompanying this change in coherence is a change in the power spectral density (PSD). There is a reshaping of power in the stimulus frequency ranges $(0-20$ and $40-60 \mathrm{~Hz})$ as well as a small decrease in the asymptotic value of the PSD, corresponding to the mean firing rate (Cox and Lewis, 1966). The coherence of burst spikes to the local signal decreases with increased global intensity, as expected. However, there is a more pronounced decrease in the mean firing rate of these spike trains (Fig. $6 c$, middle). Bursts have been considered as unitary elements of information, serving the purpose of promoting synaptic reliability (Lisman, 1997). To examine this hypothesis, we found that the coherence between the stimulus and initial burst spikes is slightly smaller than the coherence with all burst spikes ( $\sim 20 \%$ reduction for local; $\sim 15 \%$ reduction for local plus global) (supplemental Fig. 3, available at www. jneurosci.org as supplemental material), implying that spikes within a burst carry mainly redundant information. The difference, however, is consistent with observations that burst duration (Kepecs et al., 2002) and relative timing of burst spikes (Oswald et al., 2007) can encode specific stimulus features.

Our analysis in Figure 6 shows that the isolated spikes are always making a relatively minor, yet significant, contribution to the coherence to the local AM. A larger global AM even slightly enhances the low-frequency coherence of isolated spikes. This unexpected result suggests that the global high-frequency signals generated by the presence of conspecifics (Stamper et al., 2010) might actually facilitate the coding of local low-frequency signals (prey) through isolated spikes. The global AM suppresses onehalf of the bursts or more, yet the firing rate remains nearly unchanged, which means that there are more isolated spikes. These isolated spikes are able to perform temporal coding of the highfrequency signal, as measured by the high coherence values in Figure $6 c$, by riding on top of the local AM and phase locking to the high-frequency fluctuations. They can do this without diminishing their contribution to the coding of low frequencies-in fact, their relative contribution to the total low-frequency coherence increases in proportion to the intensity of the highfrequency AM.

In summary, bursts with only local AMs perform encoding because they have an internal structure not connected to the stimulus structure; but bursts also perform good temporal cod- 


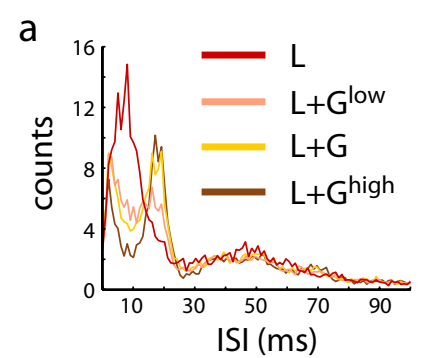

b
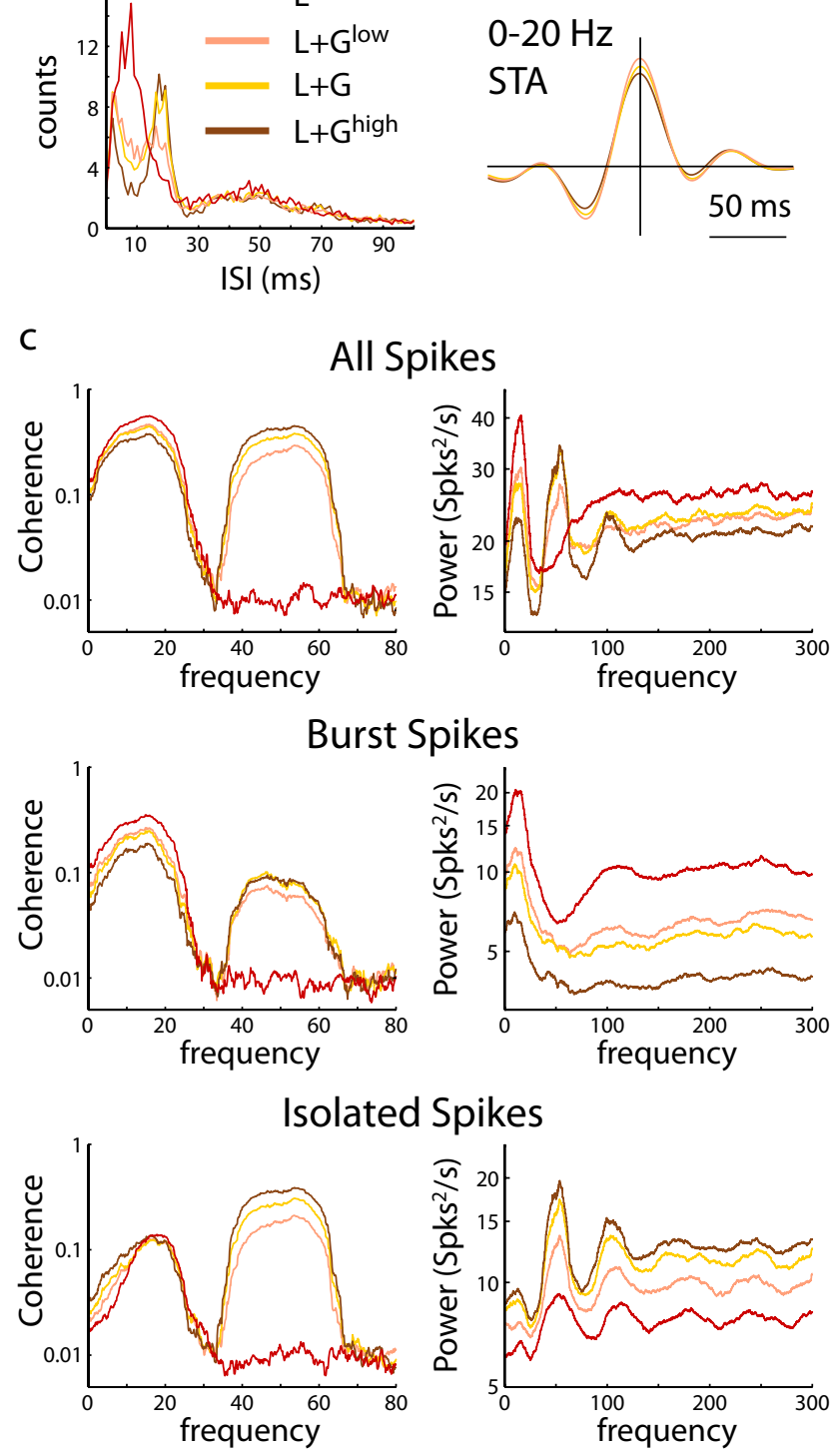

Figure 6. The proportion of low-frequency information encoded by bursts or isolated spikes depends on the strength of high-frequency sensory input. $\boldsymbol{a}$, The proportion of ISIs at small values decreases with increasing global intensity. $\boldsymbol{b}, 0$ ver these different global intensities, the STA (with respect to all spikes) of the concurrent low-frequency signal remains similar. c, Lowfrequency coherence drops with increasing high-frequency stimuli (top, left). Along with this decrease in coherence, there is also a decrease in the asymptotic power spectra (top, right). The coherence between the local stimulus component and spike trains composed only of bursts drops with global stimuli (middle, left), but there is a larger relative decrease in the asymptotic power spectrum (middle, right). The coherence between spike trains composed of isolated spikes and the local signal increases with increasing global stimulation (bottom, left). This increase in coherence is accompanied by a large relative increase in the asymptotic power spectrum (middle, right).

ing of the local AM. With local plus global, bursts again perform coding and encoding, despite their reduced numbers because of their suppression by high-frequency signals. But there are now more isolated spikes, which code mainly for the high, but also to a lesser extent for the low frequencies. The increase in isolated spikes and decrease in bursts provide the basis for the short-term plasticity-based decoding scheme described in the next section.

We tested the ability of pyramidal cell model to reproduce the stimulus-dependent statistics observed in our data. The intensity of the global stimulus was both increased and decreased and the
Model

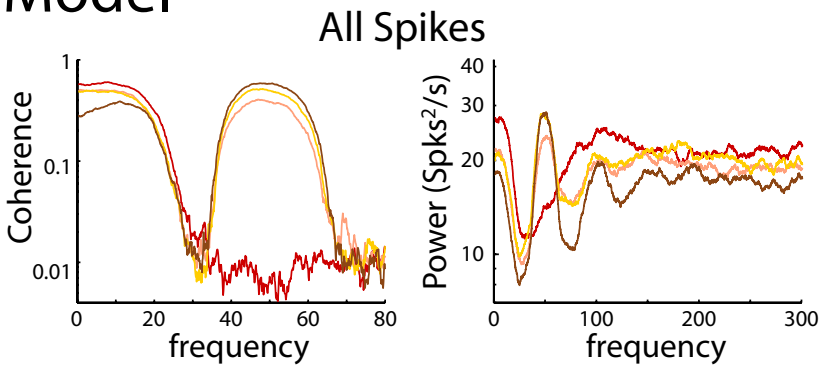

Burst Spikes

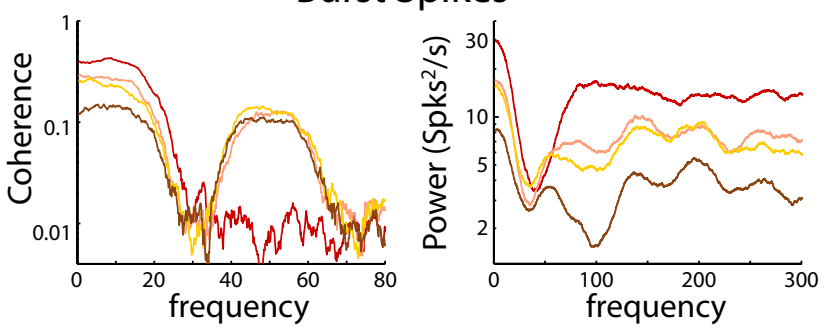

Isolated Spikes
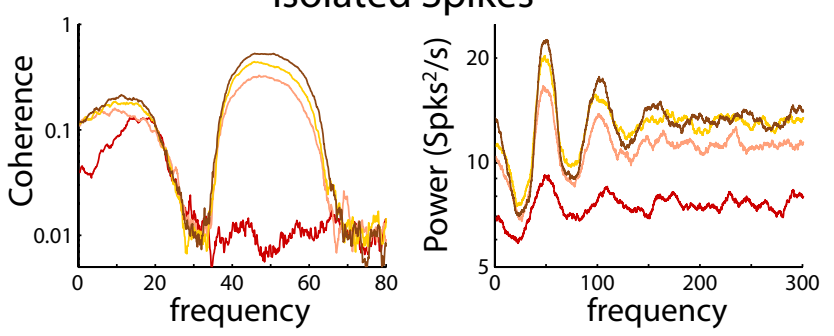

Figure 7. The pyramidal cell model successfully reproduces global intensity-dependent spike train statistics. We jointly varied global intensity and conductance pairs to mimic the responses to differing global stimulation intensity seen in Figure $6 c$. The parameter pairs used (from low to high) are $\left(\sigma_{G}, g\right)=\left(0.065 \mu \mathrm{A} / \mathrm{cm}^{2}, 0.0676 \mathrm{mS} / \mathrm{cm}^{2}\right),\left(0.09 \mu \mathrm{A} / \mathrm{cm}^{2}, 0.069 \mathrm{mS} / \mathrm{cm}^{2}\right)$, and $(0.115 \mu \mathrm{A} /$ $\mathrm{cm}^{2}, 0.071 \mathrm{mS} / \mathrm{cm}^{2}$ ). Both low-frequency coherence (top, left) and asymptotic power spectra (top, right) drop with increasing high-frequency stimuli. When burst spikes are treated separately, the low-frequency coherence drops with global stimuli (middle, left). Along with this change in coherence, there is a larger relative decrease in the asymptotic power spectrum (middle, right). The coherence between isolated spikes and the local signal increases with increasing global stimulation (bottom, left) along with a large relative increase in the asymptotic power spectrum (middle, right).

conductance was adjusted accordingly to maintain physiological firing rates. Increasing global intensity increases the coherence of the full spike train to this signal $(40-60 \mathrm{~Hz})$ while slightly decreasing the coherence to the local $(0-20 \mathrm{~Hz})$ signal (Fig. 7, top left). Both the asymptotic values of the power spectra and the relative power distribution within the stimulus frequency range (Fig. 7, top right) qualitatively match experimentally observed values (Fig. $6 c$, top right). The low-frequency coherence of the burst train exhibits a larger relative decrease (Fig. 7, middle left) with increasing global intensity as does the overall power level (Fig. 7, middle right). In contrast, the low-frequency coherence and asymptotic power spectra (indicative of mean firing rates) of the isolated spike train increase with global intensity (Fig. 7, bottom). The qualitative reproduction of higher order spiking statistics (power spectrum and coherence) by the pyramidal cell model further suggests that the phenomena we observed are primarily attributable to feedforward stimulus modulation of spiking statistics and thus do not arise from network feedback mechanisms.

The coherence of burst spike trains to the local stimulus components drops with increasing global stimulation; however, the 
a
Experiment

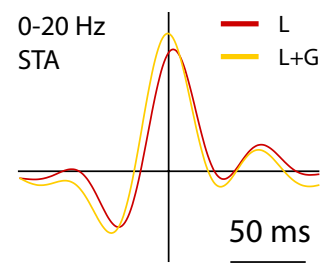

b

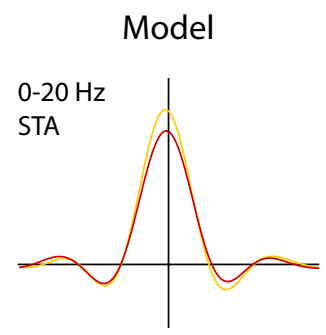

Figure 8. Encoding of stimulus features by bursts is conserved under different stimulus conditions. $\boldsymbol{a}$, The spike-triggered average (obtained using burst spikes) of the local stimulus component is very similar under local (red) and local plus global (yellow) stimulus conditions for experimental data. $\boldsymbol{b}$, The pyramidal cell model reproduces this conservation of the STA waveform. In both real and model neurons, the local STA is larger for local plus global (yellow) stimulus conditions. This effect can be understood when considering the fact that a global stimulus interferes with the burst-generating mechanisms in these cells. Only large-amplitude, low-frequency components will be able to sufficiently overcome this interference, thus contributing to a larger STA.

STA of burst spikes is relatively conserved with addition of global stimulation in both experimental (Fig. 8a) and model (Fig. 8b) data. This suggests that bursts still carry low-frequency information when global stimulation is present. However, the rate of burst emission is reduced, and therefore a postsynaptic decoding mechanism may take advantage of the different rate of bursting to distinguish these two different stimulus contexts.

Postsynaptic models with short-term plasticity models filter information carried by afferent bursts or isolated spikes Several biophysical mechanisms have been suggested as putative decoders of burst activity (Thomson, 2000b; Fortune and Rose, 2001). These mechanisms may be used to implement independent transmission of local low-frequency (prey) information to different target neural populations within TS, depending on contextual, global high-frequency (communication) sensory information.

We therefore explored potential isolated spike- versus burstdependent targeting of postsynaptic populations, motivated by experimental observations of biophysical synaptic properties of downstream ELL targets in TS (Fortune and Rose, 2000). Several cell types in the TS exhibit different forms of synaptic plasticity in response to stimulation of ELL afferent fibers, some showing short-term depression, some short-term facilitation, and others a mix of both (Fig. 9) (Fortune and Rose, 2000). To test the hypothesis that toral neurons expressing depression or facilitation will differentially mediate a sensitivity to either isolated or burst spikes, we developed a model of postsynaptic neurons with feedforward plastic synapses (see Materials and Methods).

Our model of a depressing synapse has a high baseline synaptic efficacy, such that a single spike will evoke a large postsynaptic potential (Fig. 9a, top left). A burst will progressively decrease the synaptic strength if the time constant of recovery is longer than the burst duration. As a result, each subsequent postsynaptic potential (PSP) is attenuated relative to the preceding one (Fig. 9a, top right). The converse behavior is seen for our model of a facilitating synapse-single spikes evoke a small PSP, whereas incoming bursts potentiate late PSPs (Fig. 9a, bottom).

When our postsynaptic model is driven by the pyramidal cell model spike trains, under different stimulus conditions, an interesting response dichotomy emerges. The mean firing rate of the model with synaptic depression increases as the intensity of the

\section{Postsynaptic Model}

a

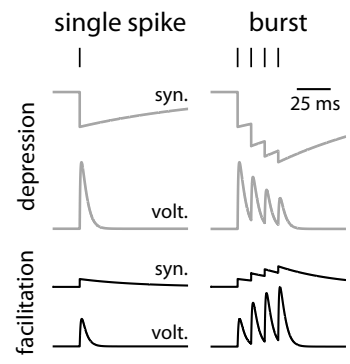

b

\section{Facilitation}

C

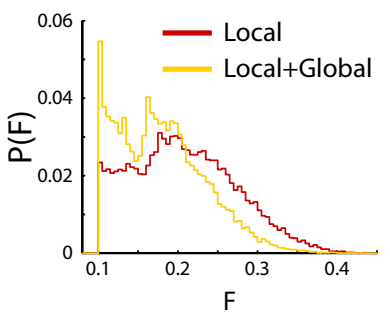

e

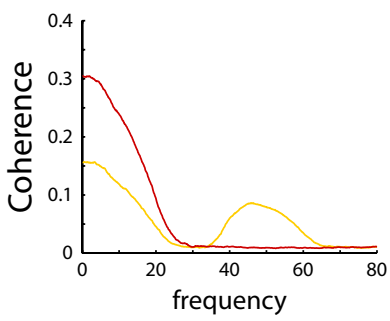

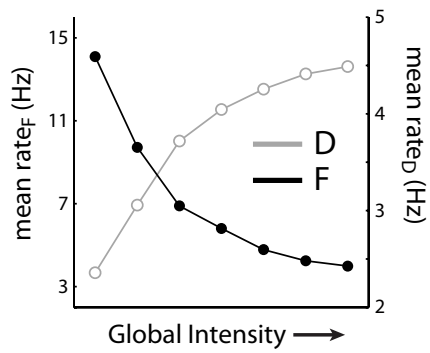

Depression

d

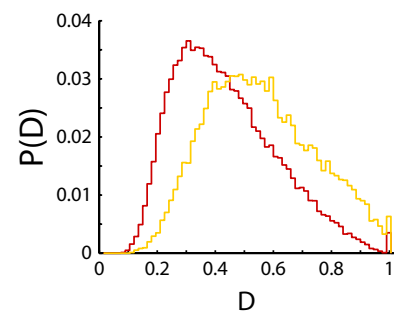

f

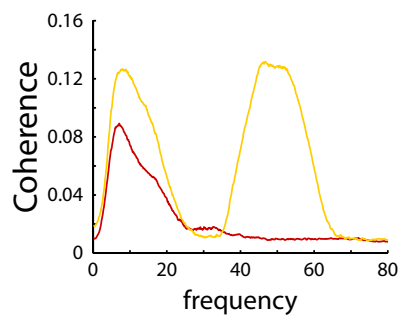

Figure 9. Synaptic facilitation or depression bias the sensitivity of postsynaptic populations to either bursts or isolated spikes, thus allowing selective transmission of different sensory stimuli. $\boldsymbol{a}$, A model of synaptic depression (gray) responds with high synaptic efficacy and a large PSP to a single, isolated spike (top left). When this model is driven with a burst, the synaptic efficacy progressively decreases and successive potentials are smaller (top right). A model of synaptic facilitation (black) responds with low synaptic efficacy and a small PSP to a single spike (bottom left). An incoming burst progressively increases efficacy and the strength of successive PSPs (bottom right). $\boldsymbol{b}$, The mean rate of postsynaptic neurons with facilitation ( $F$ ) (closed circles) monotonically decreases with increasing global intensity. The firing rate of the model with depression (D) (open circles) increases with increasing global stimulus intensity. $c$, The distribution of facilitating synaptic strength variables, at the presynaptic spike times, is biased to higher values with local stimulation (red) when compared with local plus global stimulation (yellow).d, The opposite trend occurs with depressing synapses — global plus local stimulation biases the distribution to higher values (yellow) than local stimulation does (red).e, In addition to the higher firing rates caused by local stimulation in the facilitating model ( $\boldsymbol{b})$, the low-frequency coherence between the stimulus and the postsynaptic spike train is higher under this condition (red) than when global is concurrently delivered (yellow). $\boldsymbol{f}$, The low-frequency coherence is higher with local plus global stimulation (yellow) than with only local (red) in a postsynaptic model with synaptic depression.

global stimulus component is increased (Fig. $9 b$, open circles). In contrast, the model with facilitating synapses fires less frequently as the global stimulus intensity is increased (Fig. $9 b$, closed circles). How this differential rate sensitivity arises is explained by the distributions of synaptic strengths at the times of incoming spikes. The additional burst content in pyramidal cell spike trains, caused by local stimulus, increases the relative drive to postsynaptic targets. This biases the distribution of synaptic strengths to either higher values when the synapses are facilitating (Fig. $9 c$, red) or lower values for depressing synapses (Fig. $9 d$, 
red). Concurrent global stimulation interferes with the burst mechanism and thus removes additional drive to these synapses arising from a high-frequency barrage of spikes within a burst. Under this condition, facilitating synapses will have a lower efficacy (Fig. $9 c$, yellow) relative to the local alone condition, and depressing synapses will be allowed to recover more, thus increasing their average efficacy (Fig. 9d, yellow).

Without a corresponding change in the information carried by the postsynaptic spike trains, the firing rate changes may not sufficiently form the basis of a context-dependent routing network. To examine this, we calculated the coherence between the sensory stimulus and the postsynaptic spike trains, for different synaptic models under difference stimulus conditions. The low-frequency coherence of the facilitation model decreases nearly twofold with the addition of highfrequency stimulus (Fig. 9e). This effect is consistent with the relative synaptic efficacies under these conditions. The lowfrequency coherence of the depression model, however, increases when global stimulus is added (Fig. 9f), thus increasing both the output rate and the informa-

tion carried by this pathway in this environmental context.

Here, the additional context is the presence of conspecifics, which causes additional global inputs at higher frequencies to E-cells, which in turn reduces E-cell bursting. This routing may permit electric fish to continue processing information related to prey location, via both burst (no conspecifics present) and isolated spikes (in the presence of conspecifics with very different EOD frequencies).

\section{Discussion}

Burst firing is considered an important feature of neural coding (Mainen and Sejnowski, 1995; Kepecs et al., 2002; Krahe and Gabbiani, 2004; Lesica and Stanley, 2004; Oswald et al., 2004; Schwartz et al., 2007; Lin and Nicolelis, 2008; Marsat et al., 2009). Bursts are produced through different mechanisms (Izhikevich, 2000), elicited under different stimulus conditions (Doiron et al., 2003), and code different stimulus features (Oswald et al., 2004; Marsat et al., 2009). They may be elementary units of a coding language or simply increase the efficiency of feedforward neural pathways, or both. In the visual system, lateral geniculate nucleus neurons emit both bursts and isolated spikes in response to visual stimulation, with different stimulus conditions preferentially evoking these two firing patterns (Lesica and Stanley, 2004; Alitto and Usrey, 2005; Grubb and Thompson, 2005). In the electrosensory system, burst firing is also elicited by specific stimuli and, within these stimuli, are feature specific (Oswald et al., 2004, 2007; Doiron et al., 2007). How burst firing in the ELL of weakly electric fish encodes for combinations of spatially and temporally distinct signals and how they impact higher brain centers have not been resolved.

ELL pyramidal cells are sensitive to a broad range of temporal frequencies (contained in the inputs they receive via sensory afferents), but their selectivity depends on the spatial extent of

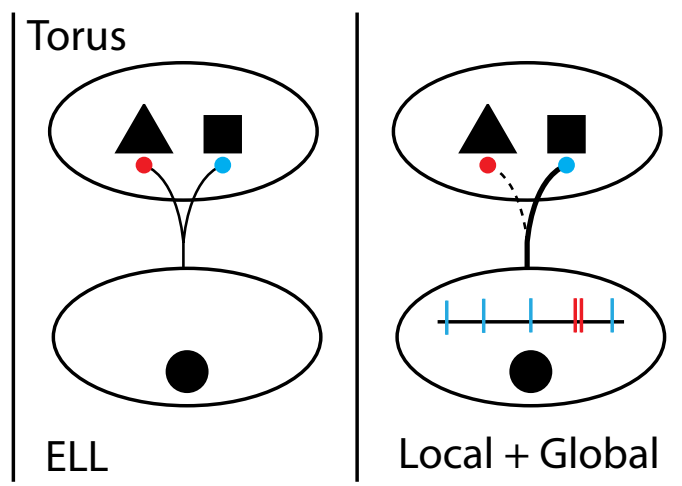

Figure 10. ELL-to-torus (TS) pathways can be differentially activated, depending on the form of plasticity in that response of our ELL-TS synaptic model to a low-frequency stimulus applied to the receptive field center of a pyramidal cilitating synapses strongly transmit the spike bursts (thick line), whereas the depressing synapses (dashed line) do not. 列

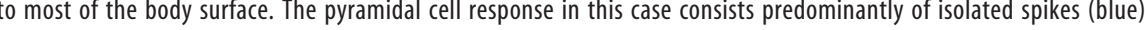
tating synapses (dashed line) do not. We therefore hypothesize that a different subset of TS neurons (square) respond to the presence of prey objects that occur when the fish is foraging in the presence of conspecifics.

stimuli. The spatiotemporal content of electrosensory modulations depends on the source of these signals. Electric fish aggregate in small groups under a wide range of behavioral and environmental contexts, including prey capture behavior, resulting in ongoing high-frequency RAMs that are global in nature (Ramcharitar et al., 2006; Stamper et al., 2010). In such complex sensory environments, the electrosensory receptor array is likely activated by simultaneous local and global stimuli. Local, slow signals arise from small environmental objects, most importantly prey, whereas global high-frequency stimuli arise from multiple EOD interactions (Middleton et al., 2006). In this study, we examined the nature of electrosensory coding under such stimulus conditions.

We recorded from ELL pyramidal cells in vivo while stimulating the electrosensory surface with simultaneous global, high-frequency and local, low-frequency RAMs. We analyzed spike trains under these different stimulus conditions and quantified neural coding performance and the prevalence of burst firing. In response to local low-frequency stimulation, E-type pyramidal cells fire bursts of action potentials whose timing is modulated by slow stimulus features. Additional high-frequency electrosensory stimuli greatly reduce burst firing. Under both of these stimulus conditions, the STA of the low-frequency stimulus component is relatively unchanged. The dependence of burst firing on combinations of local and global inputs of differing bandwidths was understood by developing an ELL pyramidal cell model with several key features. The model reproduced the effect of a dendritic compartment with somatic spike-driven, delayed backpropagating afterpotentials. The impact of these potentials was modulated by a slow potentiation variable as well as a slow, dynamic refractory period. This model was able to generate 
burst activity and reproduce its dependence on input current temporal frequency content. Slow stimulus components promoted burst firing, whereas high-frequency components led to stimulus-induced burst terminations.

Remarkably, we found that high-frequency signals actually increased power of the isolated spikes encoding the lowfrequency component of the combined signal. This suggests that electric fish might be able to detect prey efficiently in the presence or absence of conspecifics, as long as ELL downstream circuitry (TS) can successfully decode both the burst and isolated spike codes. We therefore proposed a model of downstream target neurons (Fig. 10), based on observed forms of plasticity in the torus semicircularis, which exhibit sensitivity to the level of presynaptic burst firing. Synaptic facilitation (or depression) enhances (or suppresses) firing rates in toral cell models with increased burst firing. This mechanism may serve a behaviorally relevant function by routing salient environmental information arising from local sources (e.g., from prey objects) to different downstream targets depending on electrosensory context (i.e., whether or not conspecifics were nearby). This differential routing both preserves information about prey location and may also subserve different behavioral strategies pertaining to prey capture while in groups versus isolation.

Sensory feedback plays a role in several computations performed by the ELL. Feedback from deep pyramidal cells onto intermediate and superficial pyramidal cells mediates a cancellation of self-generated movement signals (Bastian et al., 2004). Low-frequency global signals change the temporal sensitivity of pyramidal cells (Chacron et al., 2003) and render them sensitive to communication signals (Marsat et al., 2009). As only low-frequency global stimulus components are affected by this form of feedback (Chacron et al., 2003), we do not expect high-frequency global stimuli (used in this study) to affect the type of local electrosensory processing we described. As well, basilar pyramidal E-cell subtype identity may determine sensitivity to the combinations of stimuli we used in our experiments, as they exhibit differences in morphology, feedback inputs, and intrinsic membrane properties (Bastian and Courtright, 1991; Chacron et al., 2005). However, our finding that all three subtypes (superficial, intermediate, and deep) exhibit the same qualitative burst firing sensitivity to local, low-frequency and global, high-frequency signals (Fig. 3) further supports our hypothesis that the mechanism behind such processing arises primarily from the influence of feedforward sensory inputs.

The type of dynamic routing of sensory information we described may play a role in other systems. Burst firing activity has been observed in thalamocortical projection cells in auditory (He and $\mathrm{Hu}, 2002$; Massaux et al., 2004), somatosensory (Ramcharan et al., 2000; Fanselow et al., 2001), and visual system (Ramcharan et al., 2000). Burst activity is influenced by low-frequency stimulus components as well as other subtle stimulus characteristics (Lesica and Stanley, 2004; Alitto and Usrey, 2005; Grubb and Thompson, 2005), but it is not known in detail how different stimulus combinations modulate stimulus specificity. Short-term plasticity of thalamocortical synapses (Gil et al., 1999) may tune sensitivity of cortical responses to ascending sensory information relayed by modulating burst activity. However, thalamocortical synapses are predominantly depressing, whereas intracortical synapses are depressing, facilitating, or a mix of both (Gil et al., 1997). The thalamocortical pathway may simply gate burst-dependent in- formation, whereas intracortical pathways have the capacity to form a richer activity-dependent routing network for burst and isolated spike discharge in much the same way as we have proposed for the ELL-TS pathway.

Burst firing in thalamus occurs during periods of inattention while neurons fire more regularly and at higher rates during states of attention (Swadlow and Gusev, 2001). In burst mode, depressing thalamocortical synapses are allowed to recover to baseline strength during long intervals preceding bursts. Consequently, incoming bursts have a high impact at these synapses. T-type calcium currents underlying this burst mechanism are hyperpolarization activated, resulting in lower firing rates (Wang et al., 2007). This mechanism effectively forms bursts by "carving out," or removing, tonic spikes, as evidenced by the higher spontaneous rates in relay mode (Swadlow and Gusev, 2001). The mechanism we describe instead requires slow depolarizing currents to activate intrinsic dendritic feedback currents. This results in overall higher firing in burst mode, thereby eliminating excessively long silent periods preceding bursts. Subtle differences in the timing of burst spikes may also play a role in modulating synaptic efficacy depending on whether burst firing "decelerates" (as in thalamus) (Destexhe et al., 1998) or "accelerates" (as in ELL) (Noonan et al., 2003) toward burst termination. All together, the mechanism by which neurons transition from tonic to burst firing affects spike patterning and overall levels of excitability and thus dictates their impact on transmission of information to postsynaptic targets via plastic synapses. A careful comparison of the two scenarios is an interesting avenue for future studies.

Oscillatory activity is observed in many cortical areas. High-frequency cortical oscillations are believed to facilitate numerous computations. They may reduce spike count variability and increase stimulus discrimination (Masuda and Doiron, 2007), or act to synchronize subsets of neuronal populations, increasing their impact on a single postsynaptic target (Fries, 2005). Also, network high-frequency activity is dependent on attentional (Fries et al., 2008) and behavioral (Niell and Stryker, 2010) states. Changes in high-frequency network activity may also be effective at modulating cortical burst firing, thus determining the flow of information through networks with synaptic plasticity.

For electric fish, a type of coding scheme may be in place to aid in prey capture when there are potential competitors nearby. Rerouting neural information via short-term synaptic plasticity may be advantageous if the new target is part of a pathway that offers devoted processing capacity and may be more sensitive to certain stimulus features (low-frequency local information) in the presence of additional environmental demands because of the presence of conspecifics. We finally note that the presence of the high-frequency global signals attributable to conspecifics (Stamper et al., 2010) will still be signaled by ELL pyramidal neurons (Krahe et al., 2008; Marsat et al., 2009) whose receptive fields are not being stimulated by local signals.

\section{References}

Alitto HJ, Usrey WM (2005) Dynamic properties of thalamic neurons for vision. Prog Brain Res 149:83-90.

Barrett AB, van Rossum MC (2008) Optimal learning rules for discrete synapses. PLoS Comput Biol 4:e1000230.

Bastian J, Courtright J (1991) Morphological correlates of pyramidal cell 
adaptation rate in the electrosensory lateral line lobe of weakly electric fish. J Comp Physiol A 168:393-407.

Bastian J, Nguyenkim J (2001) Dendritic modulation of burst-like firing in sensory neurons. J Neurophysiol 85:10-22.

Bastian J, Chacron MJ, Maler L (2002) Receptive field organization determines pyramidal cell stimulus-encoding capability and spatial stimulus selectivity. J Neurosci 22:4577-4590.

Bastian J, Chacron MJ, Maler L (2004) Plastic and nonplastic pyramidal cells perform unique roles in a network capable of adaptive redundancy reduction. Neuron 41:767-779.

Bell CC, Maler L (2005) Central neuroanatomy of electrosensory systems in fish. Electroreception 21:68-111.

Chacron MJ, Doiron B, Maler L, Longtin A, Bastian J (2003) Non-classical receptive field mediates switch in a sensory neuron's frequency tuning. Nature 423:77-81.

Chacron MJ, Maler L, Bastian J (2005) Feedback and feedforward control of frequency tuning to naturalistic stimuli. J Neurosci 25:5521-5532.

Cox DR, Lewis PAW (1966) The statistical analysis of series of events. London: Methun.

Destexhe A, Neubig M, Ulrich D, Huguenard J (1998) Dendritic lowthreshold calcium currents in thalamic relay cells. J Neurosci 18:3574-3588.

Doiron B, Longtin A, Turner RW, Maler L (2001) Model of gamma frequency burst discharge generated by conditional backpropagation. J Neurophysiol 86:1523-1545.

Doiron B, Laing C, Longtin A, Maler L (2002) Ghostbursting: a novel neuronal burst mechanism. J Comput Neurosci 12:5-25.

Doiron B, Chacron MJ, Maler L, Longtin A, Bastian J (2003) Inhibitory feedback required for network oscillatory responses to communication but not prey stimuli. Nature 421:539-543.

Doiron B, Oswald AM, Maler L (2007) Interval coding. II. Dendritedependent mechanisms. J Neurophysiol 97:2744-2757.

Ellis LD, Mehaffey WH, Harvey-Girard E, Turner RW, Maler L, Dunn RJ (2007) SK channels provide a novel mechanism for the control of frequency tuning in electrosensory neurons. J Neurosci 27:9491-9502.

Fanselow EE, Sameshima K, Baccala LA, Nicolelis MA (2001) Thalamic bursting in rats during different awake behavioral states. Proc Natl Acad Sci U S A 98:15330-15335.

Fernandez FR, Mehaffey WH, Turner RW (2005) Dendritic $\mathrm{Na}^{+}$current inactivation can increase cell excitability by delaying a somatic depolarizing afterpotential. J Neurophysiol 94:3836-3848.

Fortune ES, Rose GJ (2000) Short-term synaptic plasticity contributes to the temporal filtering of electrosensory information. J Neurosci 20:7122-7130.

Fortune ES, Rose GJ (2001) Short-term synaptic plasticity as a temporal filter. Trends Neurosci 24:381-385.

Fries P (2005) A mechanism for cognitive dynamics: neuronal communication through neuronal coherence. Trends Cogn Sci 9:474-480.

Fries P, Womelsdorf T, Oostenveld R, Desimone R (2008) The effects of visual stimulation and selective visual attention on rhythmic neuronal synchronization in macaque area V4. J Neurosci 28:4823-4835.

Gabbiani F, Koch C (1998) Principles of spike train analysis, Ed 2. Cambridge, MA: MIT.

Gil Z, Connors BW, Amitai Y (1997) Differential regulation of neocortical synapses by neuromodulators and activity. Neuron 19:679-686.

Gil Z, Connors BW, Amitai Y (1999) Efficacy of thalamocortical and intracortical synaptic connections: quanta, innervation, and reliability. Neuron 23:385-397.

Grubb MS, Thompson ID (2005) Visual response properties of burst and tonic firing in the mouse dorsal lateral geniculate nucleus. J Neurophysiol 93:3224-3247.

He J, Hu B (2002) Differential distribution of burst and single-spike responses in auditory thalamus. J Neurophysiol 88:2152-2156.

Hitschfeld EM, Stamper SA, Vonderschen K, Fortune ES, Chacron MJ (2009) Effects of restraint and immobilization on electrosensory behaviors of weakly electric fish. ILAR J 50:361-372.

Izhikevich EM (2000) Neural excitability, spiking and bursting. Int J Bifurcat Chaos 10:1171-1266.

Kepecs A, Wang XJ, Lisman J (2002) Bursting neurons signal input slope. J Neurosci 22:9053-9062.
Knight BW (1972) Dynamics of encoding in a population of neurons. J Gen Physiol 59:734-766.

Krahe R, Gabbiani F (2004) Burst firing in sensory systems. Nat Rev Neurosci 5:13-23.

Krahe R, Bastian J, Chacron MJ (2008) Temporal processing across multiple topographic maps in the electrosensory system. J Neurophysiol 100:852-867.

Laing CR, Doiron B, Longtin A, Noonan L, Turner RW, Maler L (2003) Type I burst excitability. J Comput Neurosci 14:329-342.

Lemon N, Turner RW (2000) Conditional spike backpropagation generates burst discharge in a sensory neuron. J Neurophysiol 84:1519-1530.

Lesica NA, Stanley GB (2004) Encoding of natural scene movies by tonic and burst spikes in the lateral geniculate nucleus. J Neurosci 24:10731-10740.

Lewis JE, Maler L (2001) Neuronal population codes and the perception of object distance in weakly electric fish. J Neurosci 21:2842-2850.

Lin SC, Nicolelis MA (2008) Neuronal ensemble bursting in the basal forebrain encodes salience irrespective of valence. Neuron 59:138-149.

Lisman JE (1997) Bursts as a unit of neural information: making unreliable synapses reliable. Trends Neurosci 20:38-43.

Mainen ZF, Sejnowski TJ (1995) Reliability of spike timing in neocortical neurons. Science 268:1503-1506.

Maler L, Sas E, Johnston S, Ellis W (1991) An atlas of the brain of the electric fish Apteronotus leptorhynchus. J Chem Neuroanat 4:1-38.

Marsat G, Proville RD, Maler L (2009) Transient signals trigger synchronous bursts in an identified population of neurons. J Neurophysiol 102:714-723.

Massaux A, Dutrieux G, Cotillon-Williams N, Manunta Y, Edeline JM (2004) Auditory thalamus bursts in anesthetized and nonanesthetized states: contribution to functional properties. J Neurophysiol 91:2117-2134.

Masuda N, Doiron B (2007) Gamma oscillations of spiking neural populations enhance signal discrimination. PLoS Comput Biol 3:e236.

Matveev V, Wang XJ (2000) Differential short-term synaptic plasticity and transmission of complex spike trains: to depress or to facilitate? Cereb Cortex 10:1143-1153.

Mehta MR (2001) Neuronal dynamics of predictive coding. Neuroscientist $7: 490-495$.

Middleton JW, Longtin A, Benda J, Maler L (2006) The cellular basis for parallel neural transmission of a high-frequency stimulus and its lowfrequency envelope. Proc Natl Acad Sci U S A 103:14596-14601.

Middleton JW, Harvey-Girard E, Maler L, Longtin A (2007) Envelope gating and noise shaping in populations of noisy neurons. Phys Rev E Stat Nonlin Soft Matter Phys 75:021918.

Moore CI, Cao R (2008) The hemo-neural hypothesis: on the role of blood flow in information processing. J Neurophysiol 99:2035-2047.

Nelson ME (1994) A mechanism for neuronal gain control by descending pathways. Neural Comput 6:242-254.

Nelson ME, Maciver MA (1999) Prey capture in the weakly electric fish Apteronotus albifrons: sensory acquisition strategies and electrosensory consequences. J Exp Biol 202:1195-1203.

Niell CM, Stryker MP (2010) Modulation of visual responses by behavioral state in mouse visual cortex. Neuron 65:472-479.

Noonan L, Doiron B, Laing C, Longtin A, Turner RW (2003) A dynamic dendritic refractory period regulates burst discharge in the electrosensory lobe of weakly electric fish. J Neurosci 23:1524-1534.

Oswald AM, Chacron MJ, Doiron B, Bastian J, Maler L (2004) Parallel processing of sensory input by bursts and isolated spikes. J Neurosci 24:4351-4362.

Oswald AM, Doiron B, Maler L (2007) Interval coding. I. Burst interspike intervals as indicators of stimulus intensity. J Neurophysiol 97:2731-2743.

Panzeri S, Petersen RS, Schultz SR, Lebedev M, Diamond ME (2001) The role of spike timing in the coding of stimulus location in rat somatosensory cortex. Neuron 29:769-777.

Ramcharan EJ, Gnadt JW, Sherman SM (2000) Burst and tonic firing in thalamic cells of unanesthetized, behaving monkeys. Vis Neurosci 17:55-62.

Ramcharitar JU, Tan EW, Fortune ES (2006) Global electrosensory oscillations enhance directional responses of midbrain neurons in eigenmannia. J Neurophysiol 96:2319-2326. 
Rieke F, Warland D, de Ruyter van Steveninck RR, Bialek W (1997) Spikes: exploring the neural code. Cambridge, MA: MIT.

Saunders J, Bastian J (1984) The physiology and morphology of two types of electrosensory neurons in the weakly electric fish Apteronotus leptorhynchus. J Comp Physiol A 154:199-209.

Schwartz G, Taylor S, Fisher C, Harris R, Berry MJ 2nd (2007) Synchronized firing among retinal ganglion cells signals motion reversal. Neuron 55:958-969.

Stamper SA, Carrera-G E, Tan EW, Fugère V, Krahe R, Fortune ES (2010) Species differences in group size and electrosensory interference in weakly electric fishes: implications for electrosensory processing. Behav Brain Res 207:368-376.

Swadlow HA, Gusev AG (2001) The impact of "bursting" thalamic impulses at a neocortical synapse. Nat Neurosci 4:402-408.

Tan EW, Nizar JM, Carrera-G E, Fortune ES (2005) Electrosensory interference in naturally occurring aggregates of a species of weakly electric fish, Eigenmannia virescens. Behav Brain Res 164:83-92.
Theunissen F, Miller JP (1995) Temporal encoding in nervous systems: a rigorous definition. J Comput Neurosci 2:149-162.

Thomson AM (2000a) Facilitation, augmentation and potentiation at central synapses. Trends Neurosci 23:305-312.

Thomson AM (2000b) Molecular frequency filters at central synapses. Prog Neurobiol 62:159-196.

Trussell LO (1999) Synaptic mechanisms for coding timing in auditory neurons. Annu Rev Physiol 61:477-496.

Tsodyks M, Pawelzik K, Markram H (1998) Neural networks with dynamic synapses. Neural Comput 10:821-835.

Tuckwell HC (1988) Introduction to theoretical neurobiology: linear cable theory and dendritic structure. Cambridge, UK: Cambridge UP.

Vonderschen K, Chacron MJ (2009) Sparse coding of natural communication signals in midbrain neurons. BMC Neurosci 10:O3.

Wang X, Wei Y, Vaingankar V, Wang Q, Koepsell K, Sommer FT, Hirsch JA (2007) Feedforward excitation and inhibition evoke dual modes of firing in the cat's visual thalamus during naturalistic viewing. Neuron 55:465-478. 